Philosophy and Progress: Vols. LIII-LIV, January-June, July-December, 2013 ISSN 1607-2278 (Print), DOI : http://dx.doi.org/10.3329/pp.v53i1-2.21946

\section{A BRIEF SKETCH ON THE ORIGIN AND DEVELOPMENT OF PRAGMATICS}

\begin{abstract}
Hakim Arif*
Pragmatics-dealing with the nature of language usage or contextual meaning of language expression-passes a long history to establish its own identity in the world academic arena. Although pragmatics is now identified as one of the core branches of linguistics, it originally derives from a special branch of philosophy of language. This paper providing a brief sketch of the origin of pragmatics isolates not only its philosophical tradition but also the nature of interaction with semiotics and linguistics. Finally, it describes how pragmatics assimilated with two prominent knowledge traditions of the world maintaining two different names and identities as well.
\end{abstract}

\section{Pragmatics and philosophy of language}

The origin of pragmatic interpretation of language lies in philosophy of language - a branch of philosophy enriched with the contribution of philosophers' writings dedicated to

\footnotetext{
* Professor, Department of Linguistics, University of Dhaka Email: hakimarif@du.ac.bd
}

inquire into the nature, origin and usage of language. Though the history of philosophy of language possesses almost the entire time-span of linguistic study including ancient Indian, Greeko-Roman, Chinese and Arabian contribution to modern Chomskyan analysis, pragmatic school of linguistic thought starts mainly in the 50s of the last century. The tradition of philosophy of language comprises two very famous schools of thought-ideal school of philosophy and ordinary school of philosophy (Haung, 2007). The former school originated by the philosophers Gottlob Frege, Alfred Tarski, and Bertrand Russell tries to study the logical system of artificial intelligence, which consequently turns into the development of formal semantics - the abstract symbolic interpretation of language with formation of rules-developed later by their followers such as Richard Montague, David Donaldson and David Lewis. The latter school of thought, on the other hand, flourished at Oxford in the 1950s by J.L. Austin gives attention on natural language rather than its abstract symbolic form. Later J.L. Austin himself and H.P. Grice- a follower of Austin- developed the theory of speech acts and theory of conversational implicature respectively in this tradition. Other famous followers as well as philosophers of language of the latter tradition are Peter Strawson, John Searle and Ludwig Wittgenstein (Recanati, 2004). In their approaches to language study they were profoundly interested in eliciting various aspects of language like meaning, form and origin from the misty of symbolic logic to the straightforward scientific observation and analysis.

\section{Linguistics tradition and the pragmatic 'waste-basket'}

Pragmatics was given a metaphor as the 'waste-basket' of linguistics in middle of the twentieth century. This metaphor not only denotes a negative connotation of this discipline but 
also undermines its position in the core areas of linguistics at that time. But Mey (2001) positively considers it as the indicative of the distinct 'status' of pragmatic study in the early days, which eventually helped shape the skeleton of a new discipline called 'Pragmatics'.

The debate of 'abstract versus concrete' principle of language investigation, apart from the tradition of philosophy of language mentioned above, also continues in linguistics in 1950. In fact, the consequence of this debate and the dominance of abstract method of language study occurring at this period have been explained metaphorically in the history of linguistics. More specifically, at that time there imagined a group of linguists who were working with various aspects of languages including their abstract principles on a working table and a waste-basket putting in front of them. The nature of their working procedure, especially leaving the abstract features of language on the table as well as throwing other unsolved elements of ordinary language to the wastebasket has been envisaged in the following quotation.

By placing the investigation of the abstract, potentially universal, features of language in the centre of their work tables, linguists and philosophers of language tended to push any notes they had on everyday language use to the edges. As the tables got crowed, many of those notes on ordinary language in use began to be knocked off and ended up in the wastebasket. (Yule, 1996:6)

In fact, the metaphoric use of an wastebasket and indicating it as the worthless place for throwing notes and features of ordinary language mainly derived from the concept of Israeli philosopher and linguist Yehoshua Bar Hillel (191575) who considered semantics the 'waste-basket of syntax' (Mey, 2001). Hillel’s hypothesis demands further explanation to unveil the waste-basket metaphor of pragmatics.

\subsection{Approaching the study of language as science}

Following Hjelmslev (who got influenced Leibniz's earlier notion of 'conceptual calculus') there starts an initiative to treat linguistics not only as a science, but also an 'algebra' of language in the middle of the last century. The proponents of this endeavor explain language with formal reasoning and abstract symbolism, as usually done in mathematics and in formal logic. Supporting such a view of linguistic interpretation, Chomsky (1957) introduces his famous 'transformational-generative grammar' with universal algebraic formula that includes logical symbols and mathematical abstractions. These algebraic formulas work well to elicit the inner structure of syntactic elements of a language even without getting their meaning. While Chomsky achieves a great success in interpreting syntactic element excluding its meaning, semantics ultimately gets divorced, hence is regarded as the 'wastebasket of syntax' (Mey, 2001).

Later, following Chomsky, as there develops a trend to dispose of all semantic elements of sentence to the wastebasket, the basket gets filled to its edge. In addition, people in their daily life, use more complex phenomenon than mathematical abstractions, and frequently create innovative sentences that go beyond the imagination of linguistic and philosophical interpretations. Hence linguists and/or philosophers can neither figure out the underlying structuring rules of these sentences with their limited algebraic formula of syntax, nor explain these with the techniques of truth conditions derived from semantics. So, they drop many unsolved questions of sentences to the metaphoric basket called pragmatic wastebasket, which further becomes not-too-tidy in size and overflow (Mey, 2001). 
Hence, with a view to formulating the techniques to interpret the unsolved problems of the pragmatic wastebasket, there starts another trend of linguistic analysis initiated by a group of Chomsky's dissatisfied students notably Jerry Katz, J.R. Ross, George Lakoff in the late 1960s and 1970s (Haung, 2007). In doing so, they not only challenge Chomsky's way of interpretation, especially the abstract as well as mental entity of language, which detach it from the functional elements, but also design essential rules of languages with the help of theories of speech acts, conversational implicature derived from ordinary school of philosophy in order to interpret the elements and items of ordinary languages left into the pragmatic wastebasket by generative grammarians. Following these trend in 1970s a handsome number of linguistic research works were carried out by Charles Fillmore, Laurence Horn and Gerald Gazder where, metaphorically saying, the disorderliness of the aspects of this basket, finally, come into order.

\section{Pragmatics and Semantics: A comparison}

Like pragmatics semantics, one of the core fields linguistics, also concerns meaning of various linguistic components as its focal area of investigations. But these two associated branches of linguistics deal with meaning from totally different perspectives. (Arif 2012). While semantics incorporates sentence meaning or the grammatical meaning of a sentence, pragmatics extracts the meaning of utterance which includes both sentence and context (Grice 1975). Green et al. (1996: 250) and Kearns (2000:1) supporting Grice's proposition clearly states that-

1. ...semantics is concerned with those elements of meaning that can be directly decoded from the words of sentence itself, and pragmatics is concerned with those elements of meaning that depend on contextual information beyond the words of sentence itself and on the interpreter's inferential ability.

2. Semantics deals with the literal meaning of words and the meaning of the way they are combined, which taken together from the core of meaning, or the starting point from which the whole meaning of a particular utterance is constructed. Pragmatics deals with all the ways in which literal meaning must be refined, enriched or extended to arrive at an understanding of what a speaker meant in uttering a particular expression.

In fact, two aforesaid statements clearly identify a sharp borderline of both semantics and pragmatics, though these two human sciences commonly describe the meaning of language. Put clearly, the discipline investigated narrates the literal meaning, and the meaning achieved from the combined expression of a group of linguistic components of a language, whereas pragmatics points out speaker intended meaning modified by contextual factors and variations (Arif 2012). For example, the semantic meaning of the sentence, 'I feel cold now' indicates a statement a person catching cold due to staying in an icy place. But if someone utters this statement inside the air-cooled room, the pragmatic meaning will definitely be a request by the speaker to stop the air cooler machine or to reduce the volume of air cooler.

\section{Pragmatics: semiotic perspectives}

Charles Morris being influenced by Charles Sanders Peirce first used the term 'pragmatics' as a branch of semiotics in 1930s (Morris, 1938). Later this term was taken in linguistics for granted as a name of one of its core branches dealing with usage of language. Morris, in fact, isolates semiotics into three different branches - syntax, semantics and pragmatics. 
According to his interpretation, syntax deals with 'the formal relation of signs to one another', semantics denotes the study of 'the relations of signs to the object to which signs are applicable' and, finally, pragmatics incorporates the study of 'the relation of signs to interpreters' (Morris, 1938: 6; Levinson, 1983). Later Carnap (1942) (cited in Recanati, 2004b) makes an order of the degree of abstraction of these three branches. More elaborately, according to Carnap, syntax is the most abstract and pragmatics is the least abstract, whereas semantics belongs to these two in expressing the degree of abstraction.

In his article 'Pragmatics' Posner (1997), on the other hand, examines the Morrisian characterization of the subject matters of pragmatics in addition to leaving his own views and comments on semiotic-pragmatics. Thus, following Morris (1938) and Posner (1997) as well as some other relevant literature, we are now trying to envisage the skeleton of pragmatics from the perspective of semiotics.

Semiotics emphasizes the role of interpreter in using as well as extracting the meaning of sign in a communicative event. Such as this approach to sign interpretation by the interpreter relates pragmatics as its one of the core branches of semiotics, since the topics of pragmatics mainly focus on the views of addressees language use. Hence the roles of interpreters get an exclusive attention in the description of semiotics. According to the view of semiotic-pragmatics there appears two types of interpreters when a sign is produced intentionally. For example,

1) Interpreter as recipient responds to the sign with and interpretant, and

2) The sign producer him/herself to interpret the sign gets the response of the recipient.
Now what is the nature of interpretation of sign? According to Posner (1997) interpretation includes coding the signifier on the basis of signified. As far as the role of sign producer is concerned, this process further can be performed in two different ways. If the sign is a signifying sign, the sign producer performs a role of an encoder. This is because; performing a sign the sender or the sign producer encodes a message which needs to be decoded by the respective recipient of the addressee (See figure 1). Alongside, sign producer, in communicative sign, intends recipient to recognize his/her intentions to make a specific interpretant. Thus, in communicative sign, the sign producer and recipient are identified as sender and addressee respectively.

Morris's (1938: 6) definition of pragmatics 'the relations of signs to interpreters' itself implies the focal points of pragmatic research. But the terms and the nature of relationship entailed here encounter different types of interpretations over the time. Even Morris himself mentioning the focus of this definition indicates that signs are usually produced for the purpose of being decoded or interpreted. For example, in the figure 1 the addressee-the students of the class- decoded the message sent by the sender that they need to be silent this in noisy classroom. Carnap (1939: 4), almost at the same time, regards the scope of this discipline as the explanation of 'the sign, state and environment of a man who speaks or hears' different types of verbal sign, although Carnap later includes various non-linguistic signs and non-communicative semiosis in it. Having been influenced by Morris and Carnap different subsequent pragmaticians then consider pragmatics a part of human action that focuses on speech act as well as intentional coded signaling. Hence there starts an academic endeavor by linguists, philosophers, anthropologists, sociologists and psychologists to negatively treat pragmatics because in this 
discipline they used to study the meaning which concerns only usage of language. Posner (1997) indentifies such an intension of pragmaticians, especially Bar-Hillel (1971), Gazdar (1979), Horn (1988) who mainly belong to this approach. As pointed out-

The idea behind this approach is that semantics describes the meaning signified by signs without reference to interpreters and pragmatics has to study what interpreters do in order to make sense of this meaning in the situation they are in. (Posner, 1997: 228).

It can be affirmed, having critically observed this statement, that the aforesaid pragmaticians belonging to the school of restricted sense of pragmatics were also influenced by Morris's approach to pragmatics which only emphasizes the roles of interpreters who mainly incorporate a process of semiosis to decode the signs involved in a communicative event.

But as opposed to this group, in the academic domain, there appear semiotic-pragmaticians, especially Posner (1997) who treat pragmatics very broadly and include the following four focal points of this discipline.
a. Pragmatic process
b. Pragmatic signs
c. Pragmatic information
d. Pragmatics messages

a. Pragmatic process: In a communicative activity pragmatics process contains the central role. This process is nothing but the prime form of communication which is performed by both sender and addressee. In this process the sender with a view to sending a message to the addressee first connects a medium, chooses appropriate code including a signified which approximates the intended message as well as corresponding signifier. S/he then produces a sign vehicle as the token of this signifier. The addressee, on the other part, follows the identical process to reconstruct the intended message from the signifier. The whole process can be illustrated with the following example. Suppose, A Bengali school teacher puts her index finger vertically on lips in order to keep noisy class silent (See figure 1).

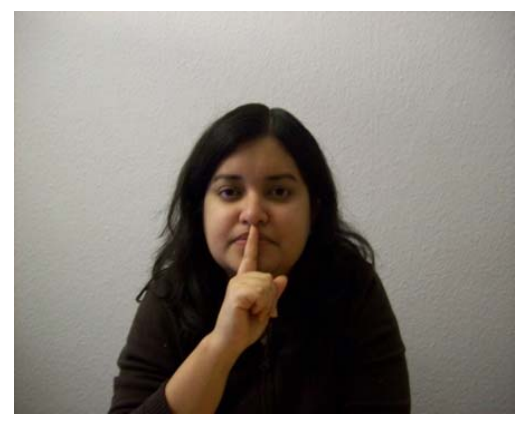

Figure 1. Showing 'keep silent' (Source: Arif 2011)

Then there appears a pragmatic process which consists of (Arif 2011)-

a. Sender: In this gesture the gesturer (gesture actor) is the sender. Here the sender could be a school teacher, the mother or father, boss of the company etc.

b. Addressee: In this gesture the addressee of the gesture is one of its recipients. Here the addressee might be students in the classroom, kids in the family, employees in the company etc.

c. Signifier: The behavior type of some person putting the index finger over the lips

d. Signified: The abstract meaning of this gesture is the signified. Here the signified is the concept of 'keep silent'. 
e. Code: Bengali cultural tradition that helps maintain a correlation between the signifier and the signified of this gesture.

$\mathrm{f}$. Message: The concrete meaning of the gesture in a given context is the message. Here the message is 'keep silent in the circumstances given'.

g. Medium: All sign factors of the sign process taken together.

h. Context: If the sender of this gesture is the class teacher and the addressees are the students, the context is the classroom.

In fact, the above sign process reflects that sender-the school teacher- containing a message-'keep silent in the circumstance given'- with a signified 'keeping the noisy class silent' in producing the gesture of putting the index finger on the lips as signifier and the sign vehicle as well. On the other hand, the sign process gets succeeded when the addresseestudents in the classroom- decodes this signifier, in reaction to the sender's gesturing, with the identical signified that the sender intends, and finally gets a message that they need to be silent in the given circumstance given.

In the contemporary literature, there exists other types of pragmatic processes that work in reverse orders (Posner, 1980). Firstly, code-related sign process which involves encoding in the sender and decoding in the addressee. Secondly, situationdepending inferencing incorporates a rather complex inference process that occur both simultaneously as well as maintaining a sequence.

b. Pragmatic sign: The aspect of pragmatic sign indicates that some signs possess the properties for which they are either regarded as simple signs, or complex signs, even partial signs. For example, in the figure 1 the simple sign is 'the behavior token of a concrete person putting the index finger over the lips'.

Sometimes such pragmatic signs are also entailed in different codes of society, especially natural language, constructed form of logic and artificial intelligence, painting style in art require complex pragmatic process (Posner, 1997). In addition, in different communication settings in our real life situation different verbal as well as non-verbal signs like personal pronouns, place and time adverbials, and pointing fingers to show the direction and others demands different additional situational indicators to interpret properly, since the nature of their identification is determined by the occurrence in time and space. Hence, Peirce in interpreting such as these examples of signs gives a special category as 'indexical' sign. All the complex or partial signs are very much included to the pragmatic process.

c. Pragmatic information: Pragmatic information is a kind of information which helps both sender and addressee to infer about the expected message in a communicative event. The sign of figure 1 mentioned above providing the sender's message 'keep silent in the circumstances given' can also be regarded as information, since the addressee living in the same linguistic context elicits the identical meaning form it. In fact, the sender is concerned with information during using special signs, whereas the addressee dependents on this information at the time of such signs interpretation. The relevant information showing above characters can be achieved from the culture of speech community of sender and addressee. More specifically, sender and addressee's social relationship, kinship relations, 
totemic relations, clan membership etc. could be more relevant in this regard. On the basis of the interpretation of indexical signs which essentially require such as these information to interpret Posner (1997) extracts three further domains of pragmatic information e.g. properties of the cotext, properties of the context, and properties of situation referred to in the communication.

d. Pragmatic message: Pragmatic message mainly relates to the substance of 'pragmatic signals' or 'pragmatic indicators' that conduces the process of interpreting of a sign involved in communicative event. In fact, pragmatic message acts as indicator which connect signified with the intended subject matter. As Posner points out-

...we propose to call only those indicators and their message "pragmatic" that contribute to the process of interpretation connect the signified of a given sign with the message intended in its production. (Posner, 1997: 234)

For example, the nonverbal sign of figure 1 raisees the message as 'keep silent in the noisy classroom'. In addition, according to Posner (1997) as the imperative "Come!” signifies the request to come, and the fact of this utterance expresses the making of this request, the pragmatic message of this imperative is "the speaker requests someone to come".

\section{Pragmatics versus Pragmalinguistics}

In the history of pragmatics there appear two different schools of thought each of which consists of different ideology, characters and scopes. These are as follows:

1. Anglo-American pragmatic thought

2. European continental pragmatic thought

\subsection{Anglo-American pragmatic thought}

Anglo-American pragmatic thought deals with systematic study of the meaning of language along with their contextual meaning variations. The main topics of investigation of this school include deixis, presupposition, conversational implicature, speech act etc., as most of the contemporary books of pragmatics incorporates nowadays. Among all aforesaid pragmatic terms and theories, since speech acts are the most widely discussed one, it would be relevant to provide brief sketch of this topic here.

Austin's (1962) general theory of speech acts developed in response to the limitation of his previous performative/constative dichotomy hypothesis. This theory emphasizes the underlying forces as well as the different senses of speech that carries out some action. In fact, Austin (1962) here unfolds a threefold distinction of speech acts which the speakers simultaneously perform when saying something. More specifically, these three acts together give an utterance a purpose. Austin indicates that every utterance amounts to a specific action and comprises three functions to make this utterance fruitful both to the speaker and the addressee. Hence an utterance needs three essential communicative functions or acts to be recognized as a speech act.

Finally, Anglo-American pragmatic school is also regarded as component view of pragmatics, since the proponents of this approach affirm that like phonetics, phonology, syntax and semantics pragmatics should be treated as the core component of language investigation (Haung, 2007). The title of this school of thought indicates its origin and development under the tradition of Anglo-American linguistic philosophy. But nowadays this approach of linguistic interpretation is very popular throughout the world. 


\subsection{European continental pragmatic thought}

European continental pragmatic thought, on the other hand, incorporates a broader aspect of linguistic pragmatic investigation, which also includes the topics of sociolinguistics, psycholinguistics and discourse analysis.

According to Prucha (1983), this school termed occasionally as pragmalinguistics is a theory of linguistic communication. It mainly explains the way of influence of communication through their verbal message. In addition, this school of thought interprets different functional perspectives of language the users perform in the form of their behavioral pattern. As Verschueren points out-

Pragmatics constitutes a general functional (i.e. cognitive, social and cultural) perspective on linguistic phenomena in relation to their usage in the form of behavior. (Verschueren, 1999: 11)

Supporting this statement this school can also be regarded as perspective view of pragmatics, as it represents a functional perspective (more likely cognitive, social and cultural) of every aspect of language behavior (Haung, 2007).

Morris's (1938) view of semiotic pragmatics considering 'the study of a huge range of psychological and sociological phenomena involved in sign system' pointed out in the earlier section is very similar to this tradition of pragmatic interpretation (Levinson, 1983: 5).

\section{Conclusion}

Pragmatics usually uncovers the contextual meanings of ordinary languages used in different everyday communicative events. The aforesaid brief sketch reveals that with a view to analyzing the underlying meaning of such these context- specific features of ordinary language pragmatics incorporates many theories, especially presupposition, speech acts, conversational implicature and so on derived from ordinary school of philosophy - a branch of philosophy of language. At the same time, since communication is exclusively a sign process performed by both sender and the receiver, pragmatics welcomes the methods semiotics frequently uses to extract the process of the signification of human communication. Finally, although pragmatics having incorporated various theories and methods from two different disciplines, it is divided into two schools of thought over the time, the component view or Anglo-American pragmatic tradition becomes prominent, hence is studied all over the world as a part of linguistic endeavor.

\section{References}

Arif, H. 2012. On some definitions of Pragmatics and Semantics. Journal of the Institute of Modern Languages, vol. 23, p.53-65

2011. Bengali Everyday Emblematic(BEE) hand gestures as communication acts: A pragmatic approach. An Unpublished $\mathrm{PhD}$ thesis. Berlin Technical University (TU, Berlin)

Austin, J, L. 1962. How to do things with words. Oxford: Oxford University Press.

Carnap, R.1939. Foundation of Logic and Mathematics. Chicago: Chicago University Press

Chomsky, N. 1957. Syntactic Structure. The Hague: Mouton

Green,D.W. and others. 1996. Cognitive Science. Blackwell Publishers

Grice, H.P.1975. Logic and Conversation. In P.Cole and J.L.Morgen(eds.) Syntax and Semantics.Vol. 3: Speech acts. Academic Press: New York

Haung, Yan. 2007. Pragmatics. Oxford University Press

Kearns, K. 2000. Semantics. Palgrave: Hampshire

Levinson, S. C. 1983. Pragmatics. Cambridge University Press 
Mey, J. L. 2001. Pragmatics An Introduction. Malden: Blackwell Publishers

Morris, C. 1938. Foundations of the theory of signs. Chicago: Oxford University Press

Posner, R.1980.Semantics and Pragmatics of sentence connectives in natural language. In Searle, J.R., Kiefer, F. and Bierwish, M.(eds.) Speech Acts Theory and Pragmatics. Reidel Publishing Company.169-204

1997.Pragmatics. In Posner, R., Robering, K., and Sebeok, T. (eds.) A Handbook on the Sign-Theoretic Foundations of Nature and Culture. Berlin and New York: Walter de Gruyter. 219-246

Prucha, J. 1983. Pragmalinguistics: East European Tradition. Amsterdam: John Benjamins Publishing

Recanati, F. 2004a. Pragmatics and Semantics. In Horn, L.R. and Ward, G. (eds.) 442-62

Verschueren, Jef. 1999. Understanding Pragmatics. London: Arnold

Yule, G.1996. Pragmatics. Oxford \& New York: Oxford University Press 